\title{
Perforated High-Grade Mixed Neuroendocrine Nonneuroendocrine Neoplasm of Cecum: Unusual Presentation of Rare Disease
}

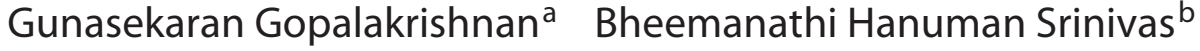 \\ Biju Pottakkat $^{a}$ Senthil Gnanasekaran ${ }^{a}$ Raja Kalayarasan ${ }^{a}$ \\ aDepartment of Surgical Gastroenterology, Jawaharlal Institute of Postgraduate Medical Education and Research, \\ Puducherry, India; 'bepartment of Pathology, Jawaharlal Institute of Postgraduate Medical Education and Research, \\ Puducherry, India
}

\section{Keywords}

Mixed neuroendocrine nonneuroendocrine neoplasm ·

Mixed adenoneuroendocrine carcinoma · Cecum •

Neuroendocrine neoplasm · Right hemicolectomy

\begin{abstract}
Mixed neuroendocrine nonneuroendocrine neoplasms (MiNENs) are rare neoplasms of the gastrointestinal tract, where the neuroendocrine as well as the nonneuroendocrine components each comprise at least $30 \%$ of the tumor. Of all cases of colorectal malignancies, MiNENs constitute around $3-9.6 \%$, with only a few cases reported to be arising in the cecum. Since majority present with nonspecific clinical and radiological findings, its diagnosis preoperatively is almost impossible and these are usually diagnosed after histopathological examination of the resected specimen. Owing to the rarity of these tumors as well as lack of complete molecular characterization, optimal treatment remains unestablished. We, here, report a rare case of MiNENs of the cecum infiltrating the right psoas muscle and presenting with perforation for which right hemicolectomy and en bloc excision of the involved psoas muscle was done followed by adjuvant chemotherapy.

(C) 2021 The Author(s)

Published by S. Karger AG, Basel
\end{abstract}

karger@karger.com www.karger.com/gat

Karger $\stackrel{\text { ' }}{5}$

BOPEN ACCESS
(C) 2021 The Author(s)

Published by S. Karger AG, Basel

This article is licensed under the Creative Commons AttributionNonCommercial-NoDerivatives 4.0 International License (CC BY NC-ND) (http://www.karger.com/Services/OpenAccessLicense) Usage and distribution for commercial purposes as well as any distribution of modified material requires written permission.

\section{Introduction}

The concurrence of an exocrine ingredient in gastrointestinal neuroendocrine neoplasms or a neuroendocrine ingredient in gastrointestinal adenomas/adenocarcinomas is not uncommon during histopathological examinations [1]. As per the WHO 2010 classification of neuroendocrine tumors (NETs) of the gastrointestinal system, these were initially divided into 3 categories as NET, neuroendocrine carcinoma (NEC), and mixed adeno-NEC (MANEC) [2].

MANECs are rare neoplasms of the gastrointestinal tract, consisting of a nonendocrine and a neuroendocrine component, each comprising at least $30 \%$ of the tumor [3]. In 2017, WHO introduced a change in the nomenclature from MANEC to mixed neuroendocrine nonneuroendocrine neoplasm (MiNEN) [4].

In medical database, only a few cases of MiNEN of cecum have been reported hitherto. We, here, report another rare case of a high-grade MiNEN of the cecum presenting with perforation. 


\section{Case Report}

A 70-year lady was referred to our outpatient department with complaints of recurrent episodes of vomiting with intermittent colicky pain abdomen for 2 months. This was associated with weight loss and loss of appetite. On abdominal examination, there was a hard mass of $8 \times 6.5 \mathrm{~cm}$ diameter in the right lower quadrant. Laboratory investigations revealed hemoglobin of $8.5 \mathrm{~g} / \mathrm{dL}$, TLC $-12,450 / \mathrm{mm}^{3}$, blood urea $-33 \mathrm{mg} / \mathrm{dL}$, serum $\mathrm{Cr}-0.6 \mathrm{mg} /$ dL, S. Na+ - 133mEq/L, S. K+ - $4.68 \mathrm{mEq} / \mathrm{L}$, and carcinoembryonic antigen (CEA) was $1.68 \mathrm{ng} / \mathrm{mL}$. Colonoscopy showed luminal narrowing, few tiny patchy ulcers, mucosal edema, and an altered vascular pattern in the cecum. Histopathological examination from the ulcers revealed edema with chronic inflammatory infiltrates in the subepithelial layer suggestive of chronic nonspecific colitis. Contrast-enhanced CT abdomen and chest revealed heterogeneously enhancing irregular circumferential wall thickening of the cecum, infiltrating the right psoas muscle with loss of fat plane with the anterolateral parietal wall (shown in Fig. 1) and few enlarged pericecal lymph nodes. There were no liver or lung metastases or evidence of any free fluid in the abdomen. She was planned for excision. A bulky growth was found arising from the cecum infiltrating the right lateral parietal wall. A perforation of size $\sim 2 \mathrm{~cm}$ was noted posteriorly into the psoas muscle. Around $50 \mathrm{~mL}$ of the purulent perilesional collection was present. A right hemicolectomy with en bloc resection of the involved psoas muscle and abdominal lavage was performed. Histopathological examination of the resected specimen revealed MANEC with an adenoma component showing high-grade dysplasia. No lymphovascular or perineural invasion was present. All 22 resected lymph nodes were free of the tumor. The adenoma component was positive for CK 20 , and the neuroendocrine component was positive for synaptophysin. Ki 67 in the neuroendocrine component was 70\%. The pathological stage was T4a N0 (shown in Fig. 2, 3). She was discharged uneventfully on a postoperative day 5 . She received $3 \mathrm{cy}-$ cles of single-agent chemotherapy (oral etoposide $50 \mathrm{mg} / \mathrm{m}^{2} /$ day for 21 days in a 28-day cycle) as adjuvant therapy after a multidisciplinary tumor board meeting given her borderline performance status (ECOG 2) and old age. Serum chromogranin A at 2 weeks was $27 \mathrm{ng} / \mathrm{mL}$, and after 6 months, $32 \mathrm{ng} / \mathrm{mL}$ with CEA levels of $1.30 \mathrm{ng} / \mathrm{mL}$. Follow-up imaging at 6 months revealed no evidence of recurrence or metastasis (shown in Fig. 4).

\section{Discussion}

Cardier in 1924 was the first to report the presence of both nonendocrine and neuroendocrine elements in gastrointestinal tumors. Lewin [5] subsequently categorized these tumors into 3 types. The composite type where neuroendocrine and nonendocrine cells are intermingled, the collision type where the neuroendocrine and nonendocrine components are distinct but abutting each other, and the amphicrine type where both components are present within the same cell. In 2010 WHO classification, the terminology "Collision" and "Composite" tumors were updated into MANECs [6].

Perforated High-Grade MiNEN of Cecum

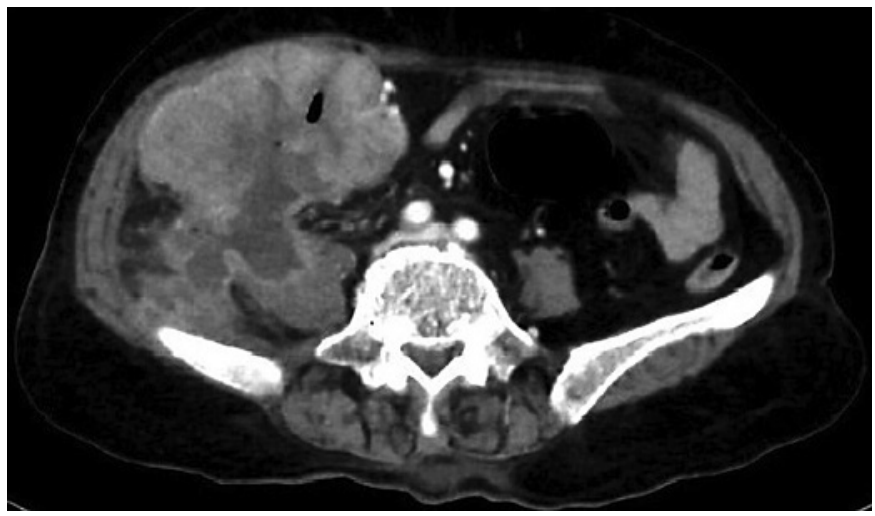

Fig. 1. CECT of abdomen showing heterogeneously enhancing irregular circumferential wall thickening of cecum infiltrating the right psoas muscle with loss of fat plane with the anterolateral parietal wall. CECT, contrast-enhanced computed tomography.

Further, in 2017, WHO changed the terminology from "mixed adenoneuroendocrine carcinoma" to "mixed neuroendocrine nonneuroendocrine neoplasm." This revision has been made to mirror that MiNENs may have a nonendocrine benign component or a malignant component other than adenocarcinomas, such as squamous cell carcinoma or acinar cell carcinoma [7]. The current 2019 WHO classification consists of 3 grades (Grades 1, 2, and 3) of NETs, while the NECs are uniformly high grade and are divided into small-cell and large-cell types (Table 1) [8].

Of all colorectal cancers, MiNENs constitute 3-9.6\%, with only a few cases reported in the cecum. The majority present with nonspecific symptoms and imaging findings and are usually detected incidentally during explorations, with diagnosis only on histopathological study [9]. In our case, the patient presented with nonspecific symptoms like vomiting, intermittent colicky pain abdomen, weight loss, and loss of appetite without any features of acute intestinal obstruction. Carcinoma cecum was suspected based on clinical features and imaging and was finally diagnosed as MiNEN after histopathological examination of the resected specimen.

MiNENs are usually small in dimensions and located either in the intramural or submucosal region. They can incorporate the mesentery and are firm in consistency resulting from desmoplasia. The mucosa overlying the lesion in the bowel may be ulcerated. Grossly, they present as polypoidal masses or as ulcerated stenotic lesions ranging from 0.5 to $14 \mathrm{~cm}$, with a mean diameter of $5 \mathrm{~cm}$ [9]. Similar to the other reported cases, our case presented with a large tumor with local extension into psoas muscle. 

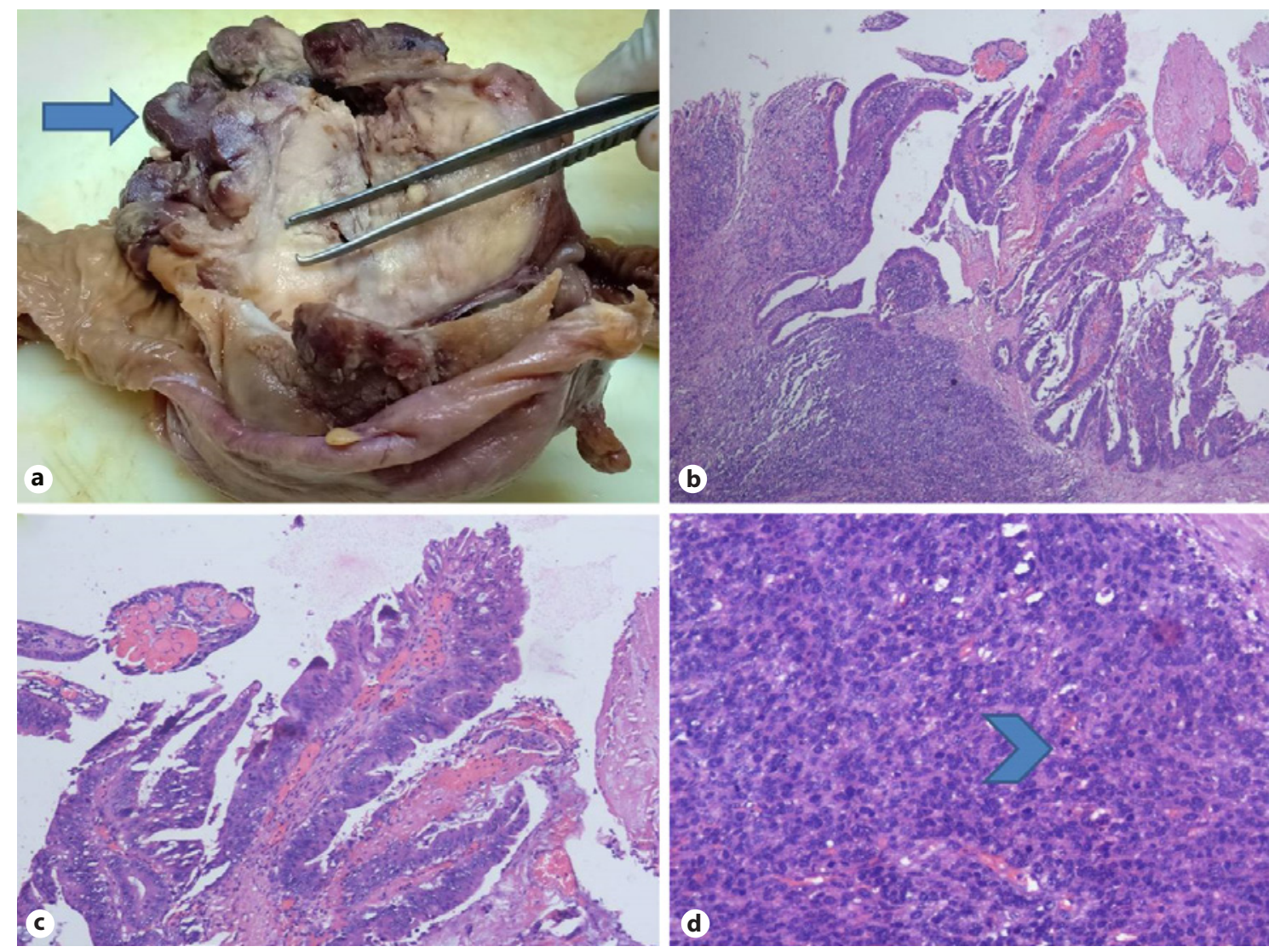

Fig. 2. Gross specimen shows evidence of mucosal hemorrhagic necrosis (arrow) and submucosal yellowish appearance (a); histopathological sections show severe dysplastic epithelium in mucosa $(\mathrm{H}$ and $\mathrm{E} . \times 100$ and $\mathrm{H}$ and E. $\times 200)($ b, c); submucosal sheets of tumor cells with oval hyperchromatic nuclei and frequent mitotic figures (arrowhead) (H and E. $\times 100$ and $\mathrm{H}$ and E. $\times 200)(\mathbf{b}, \mathbf{d})$.

Neuroendocrine neoplasms of the digestive tract are often minute and challenging to detect on radiological imaging. Both the primary lesion and its associated liver metastases augment in the arterial phase. Extension into the mesentery appears as a poorly defined lesion comprising calcification or a spiculated lesion with a stellate pattern. Mesenteric vessels when affected by desmoplastic reaction may lead to thickening and ischemia of the involved intestine [10].

Though imaging studies may raise suspicion of the lesion being NEN, a definitive diagnosis can be made only on histopathological examination. Carbohydrate antigen 125 (CA125), CEA, and carbohydrate antigen 19 (CA199) levels are usually within normal limits [11]. For diagnosis, the neuroendocrine component of MiNENs must stain positive for any 2 of chromogranin A, synaptophysin, and CD56, with the positivity for these markers being $60-70 \%, 75-90 \%$, and $50 \%$, respectively [1]. CD133 can also help diagnose these uncommon tumors by determin- ing whether the neuroendocrine component is neuroendocrine or a dedifferentiated area of adenocarcinoma [3]. In this case, as carcinoma cecum was the suspicion based on the clinical and radiological features, no other serum tumor markers were estimated preoperatively apart from CEA, as we perform only CEA for cases of carcinoma cecum in our institute.

Distant metastasis depends on tumor size, proliferation index, invasion of muscular layer, lymphovascular invasion, and perineural invasion [12]. Owing to these tumors' rarity and the lack of complete molecular characterization, optimal treatment remains unestablished. The only potentially curative treatment available is radical resection, with debulking appropriate when $>90 \%$ of the tumor can be removed. The efficacy of adjuvant chemotherapy is not unclouded in MiNENs, and the prognosis is significantly worse than that of adenocarcinoma. Because of its extremely malignant behavior, aggressive multidisciplinary management should be 

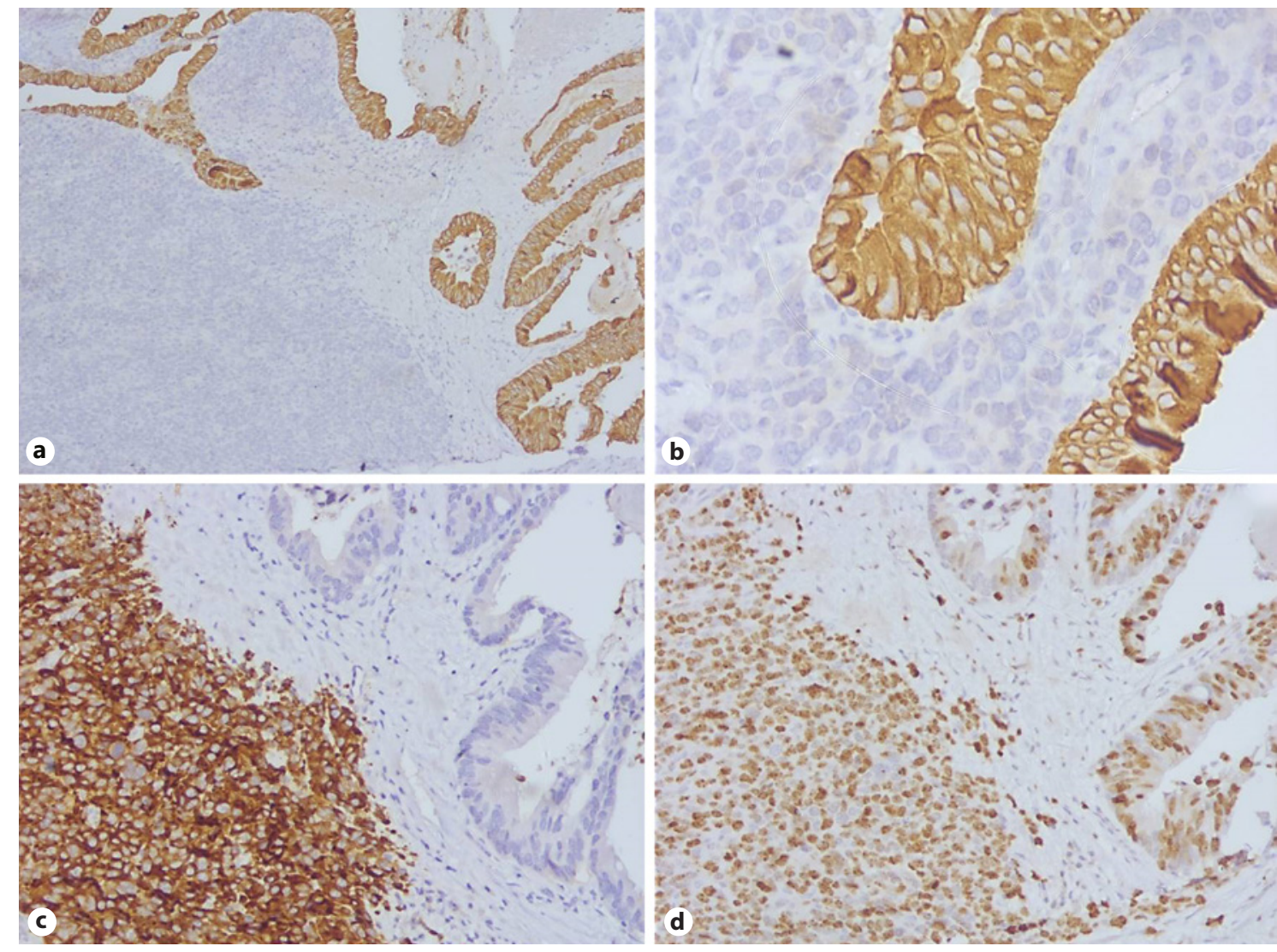

Fig. 3. IHC with pancytokeratin and CK20 show strong positive in overlying dysplastic epithelium and negative in submucosal tumor cells $(\mathrm{PanCK} \times 100$ and CK $20 \times 400)(\mathbf{a}, \mathbf{b})$; IHC with chromogranin show strong positive mainly in submucosal tumor cells and negative in overlying dysplastic epithelium (chromogranin $\times 200)(\mathbf{c}):$ High Ki 67 in both mucosal and submucosal tumor cells $(\mathrm{Ki} 67 \times 200)($ d). IHC, immunohistochemistry.

recommended including cytoreductive surgery and HIPEC [13].

Cytotoxic chemotherapy is recommended for extrapulmonary NECs, particularly colon and rectal smallcell carcinomas, akin to small-cell lung carcinoma. In a small trial using the combination cisplatin and etoposide to treat metastatic colorectal NECs, a response rate of $67 \%$ and a median survival of 19 months were attained [14]. However, the effectiveness of this regimen in the treatment of colorectal NECs is yet to be validated. According to the medical database, the median survival for patients with colorectal NECs is 5-11 months, with 3 -year and 1-year survival rates of 10 and $15 \%$, respectively [15].

Improved survival has also been seen with the FOLFOX regimen, the vincristine plus adriamycin regimen, and the carboplatin-based regimen. The use of a combination 5-fluorouracil plus oxaliplatin regimen has demonstrated improved progression-free survival compared

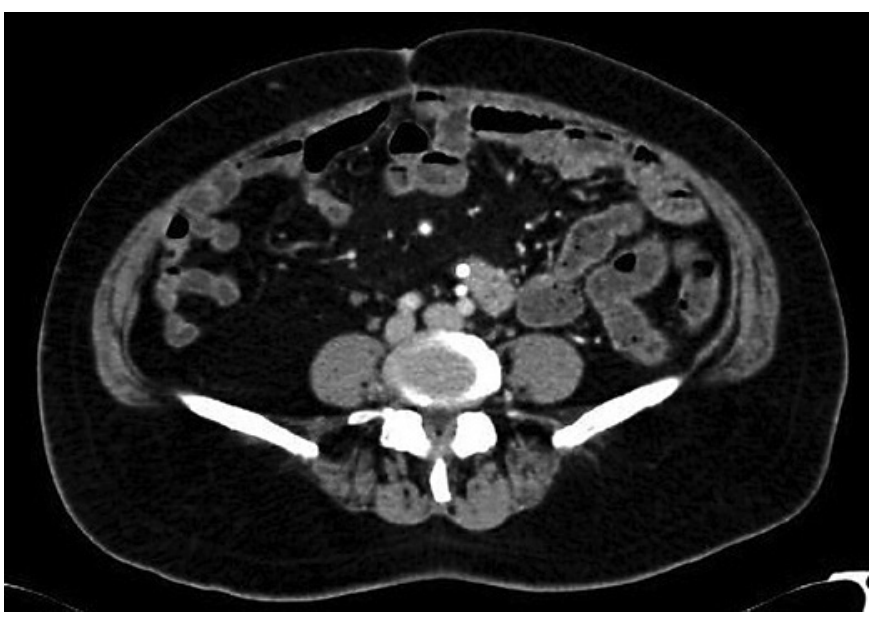

Fig. 4. Follow-up CECT of abdomen after 6 months showing no recurrence. CECT, contrast-enhanced computed tomography 
Table 1. Classification and grading criteria for NENs of the gastrointestinal tract and hepato-pancreatobiliary organs [8]

\begin{tabular}{lllll}
\hline Terminology & Differentiation & Grade & $\begin{array}{l}\text { Mitotic rate }^{\mathrm{a}} \\
(\text { mitoses/2 mm) }\end{array}$ & $\begin{array}{c}\text { Ki 67 index } \\
\text { (percent) }\end{array}$ \\
\hline NET, grade 1 & Well differentiated & Low & $<2$ & $<3$ \\
NET, grade 2 & Well differentiated & Intermediate & $2-20$ & $3-20$ \\
NET, grade 3 & Well differentiated & High & $>20$ & $>20$ \\
SCNEC & Poorly differentiated & High & $>20$ & $>20$ \\
LCNEC & Poorly differentiated & High $^{\mathrm{b}}$ & $>20$ & $>20$ \\
MiNEN & Well or poorly differentiated & Variable $^{\mathrm{c}}$ & Variable $^{\mathrm{c}}$ & Variable $^{\mathrm{c}}$ \\
\hline
\end{tabular}

NEC, neuroendocrine carcinoma; NET, neuroendocrine tumor; LCNEC, large-cell neuroendocrine carcinoma; MiNEN, mixed neuroendocrine nonneuroendocrine neoplasm; SCNEC, small-cell neuroendocrine carcinoma; NEN, neuroendocrine neoplasm. ${ }^{a}$ Mitotic rates are to be expressed as the number of mitoses $/ 2 \mathrm{~mm}^{2}$ as determined by counting in 50 fields of $0.2 \mathrm{~mm}^{2}$ (i.e., in a total area of $10 \mathrm{~mm}^{2}$ ); the Ki 67 proliferation index value is determined by counting at least 500 cells in the regions of highest labeling (hot spots), which are identified at scanning magnification; the final grade is based on whichever of the 2 proliferation indexes places the neoplasm in the higher grade category. ${ }^{b}$ Poorly differentiated NECs are not formally graded but are considered high-grade by definition. ${ }^{\mathrm{C}}$ In most MiNENs, both the neuroendocrine and non-neuroendocrine components are poorly differentiated, and the neuroendocrine component has proliferation indexes in the same range as other NECs, but this conceptual category allows for the possibility that one or both components may be well differentiated; when feasible, each component should, therefore, be graded separately.

to 5-fluorouracil alone therapy in conditions where the metastatic lesion principally has an adenocarcinoma component [16].

Customarily, the more aggressive component of the MiNENs must be considered before deciding the chemotherapy. MiNENs comprising a well-differentiated neuroendocrine component and adenocarcinoma component should be treated like adenocarcinoma, while those comprising a poorly differentiated neuroendocrine component should be treated just as poorly differentiated NECs [17].

In conclusion, MiNEN of the cecum is a rare neoplasm, and only a few cases have been reported in the medical database. It needs comprehensive reporting and analysis of data of these tumors, including presentation, diagnosis, and response to therapy to devise an appropriate treatment plan.

\section{Acknowledgements}

We are grateful to the residents and staff of Department of Pathology, Medical Oncology, and Surgical Gastroenterology for their support.

\section{Statement of Ethics}

This research was conducted ethically in accordance with the World Medical Association Declaration of Helsinki. The authors have no ethical conflicts to disclose. The subject in the case report has given the consent to use details including the images for the publication.

\section{Conflict of Interest Statement}

The authors have no conflicts of interest to declare.

\section{Funding Sources}

The authors did not receive any funding sources.

\section{Author Contributions}

Dr. Gunasekaran Gopalakrishnan and Prof Dr. Biju Pottakkat: literature search, writing, and follow-up. Assist. Prof Dr. Senthil Gnanasekaran and Assoc. Prof Dr. Raja Kalayarasan: decision of treatment plan and managing treatment and correcting writing and follow-up. Assoc. Prof Dr. Bheemanathi Hanuman Srinivas: histopathological confirmation with immunohistochemistry. 


\section{References}

1 La Rosa S, Marando A, Sessa F, Capella C. Mixed adenoneuroendocrine carcinomas (MANECs) of the gastrointestinal tract: an update. Cancers. 2012 Jan;4(1):11-30.

2 Bosman FT, Carneiro F, Hruban RH, Theise ND. WHO classification of tumours of the digestive system. World Health Organization; 2010.

3 Gurzu S, Kadar Z, Bara T, Bara T, Tamasi A, Azamfirei L, et al. Mixed adenoneuroendocrine carcinoma of gastrointestinal tract: report of two cases. World J Gastroenterol. 2015 Jan 28;21(4):1329.

4 de Mestier L, Cros J, Neuzillet C, Hentic O, Egal A, Muller N, et al. Digestive system mixed neuroendocrine-non-neuroendocrine neoplasms. Neuroendocrinology. 2017; 105(4):412-25.

5 Lewin K. Carcinoid tumors and the mixed (composite) glandular-endocrine cell carcinomas. Am J Surg Pathol. 1987;11:S71-S86.

6 Qiu S, Pellino G, Warren OJ, Mills S, Goldin $\mathrm{R}$, Kontovounisios C, et al. Mixed adenoneuroendocrine carcinoma of the colon and rectum. Acta Chir Belg. 2018 Oct;118(5):273-7.
7 Choe J, Kim KW, Kim HJ, Kim DW, Kim KP, Hong S-M, et al. What is new in the 2017 World Health Organization classification and 8th American Joint Committee on Cancer staging system for pancreatic neuroendocrine neoplasms? Korean J Radiol. 2019 Jan 1;20(1): 5-17.

8 Nagtegaal ID, Odze RD, Klimstra D, Paradis V, Rugge M, Schirmacher P, et al. The 2019 WHO classification of tumours of the digestive system. Histopathology. 2020;76(2):182-

9 Jain A, Singla S, Jagdeesh KS, Vishnumurthy HY. Mixed adenoneuroendocrine carcinoma of cecum: a rareentity. J Clin Imaging Sci. 2013;3:10.

10 Horton KM, Kamel I, Hofmann L, Fishman EK. Carcinoid tumors of the small bowel: a multitechnique imaging approach. AJR Am J Roentgenol. 2004 Mar; 182(3):559-67.

11 Düzköylü Y, Aras O, Bostancı EB, Temuçin TK, Ulaş M. Mixed adeno-neuroendocrine carcinoma; case series of ten patients with review of the literature. Balkan Med J. 2018 May;35(3):263.
12 Vijayakumar S, Jacob S, Badhe B, Srinivas B. Mixed adenoneuroendocrine carcinoma: a report of four cases from South India. J Health Res Rev. 2019 Jan 1;6(1):31.

13 Carboni F, Valle M, Russo A. Mixed adenoneuroendocrine carcinoma of the cecum. Clin Res Hepatol Gastroenterol. 2019 Nov; 43(6):627.

14 Saclarides TJ, Szeluga D, Staren ED. Neuroendocrine cancers of the colon and rectum. Results of a ten-year experience. Dis Colon Rectum. 1994 Jul 1;37(7):635.

15 Bernick PE, Klimstra DS, Shia J, Minsky B, Saltz L, Shi W, et al. Neuroendocrine carcinomas of the colon and rectum. Dis Colon Rectum. 2004 Feb 1;47(2):163-9.

16 Tanaka T, Kaneko M, Nozawa H, Emoto S, Murono K, Otani K, et al. Diagnosis, assessment, and therapeutic strategy for colorectal mixed adenoneuroendocrine carcinoma. Neuroendocrinology. 2017;105(4):426-34.

17 Hervieu V, Scoazec JY. [Mixed endocrine tumors]. Ann Pathol. 2005;25(6):511-28. 